\title{
Extracellular Monomeric Tau Is Internalized by Astrocytes
}

\author{
Juan Ramón Perea ${ }^{1,2}$, Esther López ${ }^{1}$, José Carlos Díez-Ballesteros ${ }^{3}$, Jesús Ávila ${ }^{1,2}$, \\ Félix Hernández ${ }^{1,2}$ and Marta Bolós ${ }^{1,2 *}$
}

' Department of Molecular Neuropathology, Centre for Molecular Biology "Severo Ochoa", CSIC, Madrid, Spain, ${ }^{2}$ Network Center for Biomedical Research on Neurodegenerative Diseases, Madrid, Spain, ${ }^{3}$ Department of Biology and Chemistry, Alcalá University, Madrid, Spain

\section{OPEN ACCESS}

Edited by:

Diane Hanger,

King's College London,

United Kingdom

Reviewed by:

Monika Zilkova,

Institute of Neuroimmunology (SAS),

Slovakia

Roberto Piacentini,

Catholic University of the Sacred

Heart, Italy

*Correspondence:

Marta Bolós

mbolos@cbm.csic.es

Received: 20 December 2018 Accepted: 17 April 2019

Published: 01 May 2019

Citation:

Perea JR, López E,

Díez-Ballesteros JC, Ávila J,

Hernández F and Bolós M (2019)

Extracellular Monomeric Tau Is

Internalized by Astrocytes.

Front. Neurosci. 13:442.

doi: 10.3389/fnins.2019.00442
Tau is a microtubule-associated protein that is expressed in neurons. However, in a group of neurodegenerative diseases named tauopathies - characterized by an increase in aggregated and/or hyperphosphorylated Tau - the protein accumulates inside other cells, such as astrocytes and microglia. Given that these glial cells do not produce Tau, its presence can be explained by internalization from the extracellular medium and consequent formation of Tau aggregates. Among internalization mechanisms, heparan sulfate proteoglycans (HSPGs) have been proposed to be responsible for fibrillary Tau uptake in various cell types. Here we studied whether monomeric Tau, which has been observed to be internalized by glial cells such as microglia, was also taken up by astrocytes. Although this Tau form was internalized from the extracellular medium by these cells, the mechanism of uptake was found to be independent of HSPGs.

Keywords: Tau, tauopathies, astrocytes, heparan sulfate proteoglycans, internalization, propagation

\section{INTRODUCTION}

Microtubule-associated protein Tau is expressed in neurons in the central nervous system (CNS) (Weingarten et al., 1975; Drubin and Kirschner, 1986). The abnormal aggregation and hyperphosphorylation of Tau accumulated within the cells is a common pathological hallmark of a group of neurodegenerative diseases named tauopathies (Spires-Jones et al., 2009; Medina et al., 2016). In this regard, the accumulation of this pathological and toxic Tau species in glial cells, specifically microglia and astrocytes, is described in several tauopathies (Buee and Delacourte, 1999; Kovacs et al., 2016; Ferrer et al., 2018). Given that glial cells do not contain endogenous Tau, the presence of this protein in this cell type can only be explained by its internalization from the extracellular milieu. In Alzheimer's disease (AD), the most prevalent tauopathy, and Tau propagates following a stereotypical pattern (Braak and Braak, 1991). In the context of this disease, Tau is secreted to the medium and this extracellular form of the protein is taken up by a range of cells, including neurons, and glial cells (Gomez-Ramos et al., 2006; Calafate et al., 2015). Consequently, this internalization contributes to the spread of Tau from cell to cell (Holmes and Diamond, 2014; Hyman, 2014; Medina and Avila, 2014b).

Tau can be internalized by glial cells through several mechanisms (Leyns and Holtzman, 2017). In this regard, Tau aggregates are taken up by cultured cells, primary neurons, and brain through heparan sulfate proteoglycans (HSPGs) (Holmes et al., 2013; Stopschinski et al., 2018; Yamada and Hamaguchi, 2018). Furthermore, Tau fibril species are internalized in astrocytes via the lysosomal pathway (Martini-Stoica et al., 2018). These and other observations thus indicate that aggregated Tau is directly related to the progression of tauopathies. However, because monomeric Tau is 
considered less pathogenic, its internalization, and role in the progression of these diseases have received little attention. We have previously reported that monomeric Tau is internalized by microglia through a specific receptor, namely CX3CR1 (Bolos et al., 2017a). Furthermore, Tau triggers a proinflammatory profile through p38 MAPK in these cells, thereby contributing to the progression of neurodegeneration (Perea et al., 2018a). However, the uptake of monomeric Tau in astrocytes remains unknown.

To increase knowledge of Tau propagation mechanisms, and based on previous studies highlighting the role of HSPGs in the uptake of fibrillary Tau, here we addressed the involvement of HSPGs in the internalization of monomeric Tau in primary astrocyte cultures.

\section{MATERIALS AND METHODS}

\section{Animals}

Wistar rats were used for the astrocyte primary culture experiments. The animals were obtained from the CBMSO (Centro de Biología Molecular Severo Ochoa) animal facility and were housed in a specific pathogen-free colony facility, in accordance with European Community Guidelines (directive 86/609/EEC), and handled following European and local animal care protocols (AEEC-CBMSO-62/14).

\section{Primary Cultures}

Astrocytes were cultured from the cerebral cortices of P0-P3 rats. Cortices were dissected, stripped of meninges, and digested with $0.1 \%$ trypsin at $37^{\circ} \mathrm{C}$ in HBSS (Gibco, United States) medium for 10 min. Trypsinization was stopped by the addition of DMEM culture medium supplemented with $10 \%$ fetal bovine serum (Gibco, United States), $100 \mathrm{U} / \mathrm{mL}$ of penicillin, and $0.1 \mathrm{mg} / \mathrm{mL}$ of streptomycin (Gibco, United States). A single-cell suspension of the digested tissue was obtained by repeated pipetting. Cells were seeded into a $75-\mathrm{ml}$ flask and cultured in medium at $37^{\circ} \mathrm{C}$ in humidified $5 \% \mathrm{CO}_{2}-95 \%$ air for 10 days. They were then seeded onto 12 -well plates $\left(2 \times 10^{5}\right.$ cells/well). Adherent cells were incubated for $72 \mathrm{~h}$ before being used for the experiments. For immunocytochemistry experiments, the cells were seeded onto 24 -well plates $\left(1 \times 10^{5}\right.$ cells/well $)$ with coverslips.

\section{Human Monomeric Tau}

The recombinant human Tau isoform, containing 2 N-terminal inserts, and 4 microtubule binding repeats (Avila et al., 2016), was isolated as previously described (Medina et al., 1995; Perea et al., 2018a). One $\mathrm{mg} / \mathrm{ml}$ of purified Tau was labeled with sulfoindocyanine Cy5 dye (GE Healthcare, United Kingdom) as described previously (Gomez-Ramos et al., 2009) and following the manufacturer's recommendations.

\section{Tau Internalization Assay}

Primary astrocyte cultures were treated with either $1 \mu \mathrm{M}$ of TauCy5 or PBS-Cy5 for between 0 to $24 \mathrm{~h}$. In the case of heparin or heparinase experiments, cultures were pre-treated with $20 \mu \mathrm{g} / \mathrm{ml}$ of heparin (Sigma, United States) or $10 \mathrm{mU} / \mathrm{ml}$ of heparinase III
(Sigma, United States) for 3 and $2 \mathrm{~h}$, respectively before treatment with Tau-Cy5 or PBS-Cy5, as described above. Afterward, the cells were washed three times with PBS in order to remove excess Tau attached to the membrane. They were then prepared for immunocytochemistry or immunoblotting analysis.

\section{Chinese Hamster Ovary (CHO) Cell Line}

$1.2 \times 10^{5}$ cells/well were plated in a 24 -well plate with coverslips. After $24 \mathrm{~h}$, cells were treated with heparinase at 10 and $100 \mathrm{mU} / \mathrm{ml}$ or PBS, in the case of control conditions, for $2 \mathrm{~h}$. Afterward, the cells were washed three times with PBS and prepared for immunocytochemistry as described below.

\section{Immunocytochemistry}

After treatment, primary, culture and CHO cells were fixed in 4\% paraformaldehyde in PBS for $15 \mathrm{~min}$ at room temperature (RT) and washed three times in PBS. Cells were then permeabilized with PBS containing $0.2 \%$ Triton X-100 for $10 \mathrm{~min}$ at RT and washed three times in PBS. After $30 \mathrm{~min}$ of blocking with $1 \%$ bovine serum albumin (BSA) in PBS containing $0,1 \%$ Tween20, the astrocytic cells were stained with rabbit antiGFAP (1:1000, Synaptic Systems, Germany), and mouse antiCy5 (1:2000, Abcam, United Kingdom). CHO cells were stained with mouse anti-HSPG 10E4 (1:100, AMSbio, United Kingdom). All antibodies were diluted in blocking solution and incubated overnight at $4^{\circ} \mathrm{C}$. The next day, cells were washed three times in PBS and then incubated for $1 \mathrm{~h}$ at RT with a donkey antirabbit IgG conjugated to Alexa Fluor 488 and donkey anti-mouse IgG conjugated to Alexa Fluor 647 (1:1000, Thermo Fisher Scientific, United States) for astrocytes, and donkey anti-mouse I gG conjugated to Alexa Fluor 488 (1:1000, Thermo Fisher Scientific, United States) for CHO cells. Finally, cells were washed three times in PBS, nuclei were labeled with DAPI (1:5000, Merck, Germany) for $10 \mathrm{~min}$ and rinsed with PBS.

\section{Confocal Image Acquisition and Image Analysis}

Confocal stacks of images were obtained in a Zeiss LSM800 confocal microscope under $25 \times$ and $63 \times$ oil objectives. For the $25 \times$ objective the numerical aperture was 0.8 , the interval between stacks: $670 \mathrm{~nm}$ and the size of the smallest pixel: $312 \mathrm{~nm} \times 312 \mathrm{~nm}$. In the case of $63 \times$ objective the numerical aperture was 1.4 , the interval between stacks: $300 \mathrm{~nm}$ and the size of the smallest pixel: $132 \mathrm{~nm} \times 132 \mathrm{~nm}$. The quantification of the images was carried out on the maximum projection images using the $25 \times$ objective. Representative images were obtained with a $63 \times$ objective. Images were analyzed using ImageJ Version $1.50 \mathrm{i}$ (National Institutes of Health, United States).

Fluorescence intensity analysis: internalized Tau-Cy5 was measured by quantifying the levels of Cy5 staining inside cells. To this end, the contour of individual cells was drawn in the GFAP channel and the total area was measured. Images were subjected to an invariant threshold and the $\mathrm{Cy} 5^{+}$area above the threshold was measured and then divided by the selected area of the cell. To determine the amount of HSPGs in CHO cells, images were subjected to an invariant threshold and the $\mathrm{HSPG}^{+}$area above 
the threshold was measured and then divided by the selected area of the cell. Data relative to fluorescence intensity are thus presented as \% of positive area. 150 astrocytes or $50 \mathrm{CHO}$ cells were analyzed for each experimental condition and time point.

\section{Immunoblotting}

The levels of Tau and $\beta$-actin in primary astrocyte cultures were determined by western blotting. After treatments, cells were lysed in RIPA buffer [50 mM Tris-HCl, pH 7.4, $150 \mathrm{mM}$ $\mathrm{NaCl}, 1 \%$ Triton X-100; 0.5\% Sodium Deoxycholate, 0.1\% SDS, and protease inhibitor mixture (Roche, Switzerland)]. Proteins were then separated on $10 \%$ sodium dodecyl sulfatepolyacrylamide gels in reducing conditions before being transferred electrophoretically onto nitrocellulose membranes (GE healthcare, United Kingdom). Membranes were blocked for $2 \mathrm{~h}$ with $5 \%$ in BSA in $50 \mathrm{mM}$ of Tris-buffered saline, $\mathrm{pH} 8$, containing $0.05 \%$ of Tween 20 (TBS-Tween). They were then incubated overnight at $4^{\circ} \mathrm{C}$ with the following antibodies: mouse anti-Tau5 antibody (1:1000, Merck, Germany); mouse anti-Cy5 antibody (1:1000, Abcam, United Kingdom); or mouse anti- $\beta$-actin antibody (1:1000, Sigma, United States). Protein expression was detected using HRP-conjugated secondary antibodies (1:2000, Agilent, United States). For quantification of immunoreactivity, images of blots were analyzed using ImageJ Version 1.50i (National Institutes of Health, United States). All results were obtained in at least three independent experiments.

\section{Statistical Analysis}

Statistical analysis was performed with GraphPad Prism software, Version 7.0a (United States). Data were tested by two-way analysis of variance. Post-hoc comparisons were analyzed using Holm-Sidak's or Tukey's test. Differences were considered statistically significant when the probability, $p$, of the null hypothesis was $\leq 0.05$. Data are presented as the means $\pm \mathrm{SE}$.

\section{RESULTS}

\section{Astrocytes Internalized Monomeric Tau From the Extracellular Medium}

The internalization of Tau fibrils into astrocytes has been described (Martini-Stoica et al., 2018). However, the uptake of monomeric Tau, which has a key role in tauopathies (Fox et al., 2011; Gerson et al., 2014; Bolos et al., 2017b), by these cells remains unknown. In this regard, here we studied the internalization of human monomeric Tau (Tau) in astrocyte primary cultures at time points ranging from 0 to $24 \mathrm{~h}$ (Figure 1). To facilitate the detection of Tau inside the cells by immunocytochemistry, the protein was labeled with Cy5 (TauCy5), as previously described (Bolos et al., 2015). PBS-Cy5 was used as a control. The amount of Cy5 (in red) internalized (GFAP, green color) was measured at different times (Figures 1A-G). At time 0 h, no Cy5 was detected inside the cells (Figure 1A). However, as early as $30 \mathrm{~min}$, Cy5 staining, denoting Tau presence, was observed and increased over time until $24 \mathrm{~h}$, when seems to reach a plateau (Figures $\mathbf{1 B}-\mathbf{G})$.
To corroborate that the Tau detected in the astrocytes derived from the extracellular medium rather the cells themselves, the cells were treated with Tau-Cy5, or control (PBS-Cy5). The amount of Tau internalized was then analyzed in cell lysates by western blot using Tau5, a Tau antibody (Figure 1H). The quantification is shown in Figure 1F. The presence of Tau protein was not detected at early times, thereby supporting the notion that the source of Tau is extracellular (Figure 1H). However, after $1 \mathrm{~h}$ and until $24 \mathrm{~h}$, Tau increased inside the cells compared to controls (Figures 1H,I).

These results confirm that Tau in astrocytes derives from the extracellular medium and that its internalization increases over time.

\section{Heparan Sulfate Proteoglycans (HSPGs) Are Not Involved in the Internalization of Monomeric Tau}

The internalization of Tau in aggregate and fibrillary forms through HSPGs has been studied using various cell models (Holmes et al., 2013; Martini-Stoica et al., 2018). However, the implication of these structures in the internalization of monomeric Tau has been addressed only in neurons (Katsinelos et al., 2018; Rauch et al., 2018). Hence, following the same protocol described in previous studies (Ihse et al., 2017), here we studied the uptake of monomeric Tau by astrocytes through HSPGs (Figure 2). Using primary cultures of astrocytes, the internalization of Tau was analyzed at different times in the presence or absence of heparin (Figures 2A,B). Heparin can be used to competitively inhibit binding to HSPGs and prevent Tau uptake via these structures (Holmes et al., 2013). After 1 and $3 \mathrm{~h}$ of heparin treatment, Tau protein was detected inside the cells independently of the presence of heparin (Figures 2A,B). In addition, the amount of Tau in primary cultures treated with heparinase, which removes HSPGs (Figures 2C,D), was measured. After $1 \mathrm{~h}$, Tau was found inside the astrocytes that were treated with heparinase, in the same way as those not treated. These results were confirmed using immunocytochemistry approach (Figures 2E,F). The quantification (Figure 2E) and the representative images after $1 \mathrm{~h}$ of Tau-Cy5 treatment with or without heparin and heparinase (Figure 2F), confirm that the amount of Tau inside the cells were the same. In order to be sure that HSPGs were removed properly, $\mathrm{CHO}$ cells were treated with 0 (control), 10, or $100 \mathrm{mU} / \mathrm{ml}$ of heparinase for $2 \mathrm{~h}$ (Figures 2G,H). The representative images (Figure 2G) and quantification (Figure 2H) confirm that the amount of HSPGs was reduced after heparinase treatment. As a control, the total area of the cells was measured after the heparinase treatment and no changes were observed (Figure 2I). These results therefore suggest that monomeric Tau is internalized by these cells through a mechanism that is not mediated by HSPGs.

\section{DISCUSSION}

Tauopathies are a group of disorders characterized by the abnormal accumulation of the microtubule-associated protein Tau (Goedert and Spillantini, 2011). In tauopathies such as AD, 

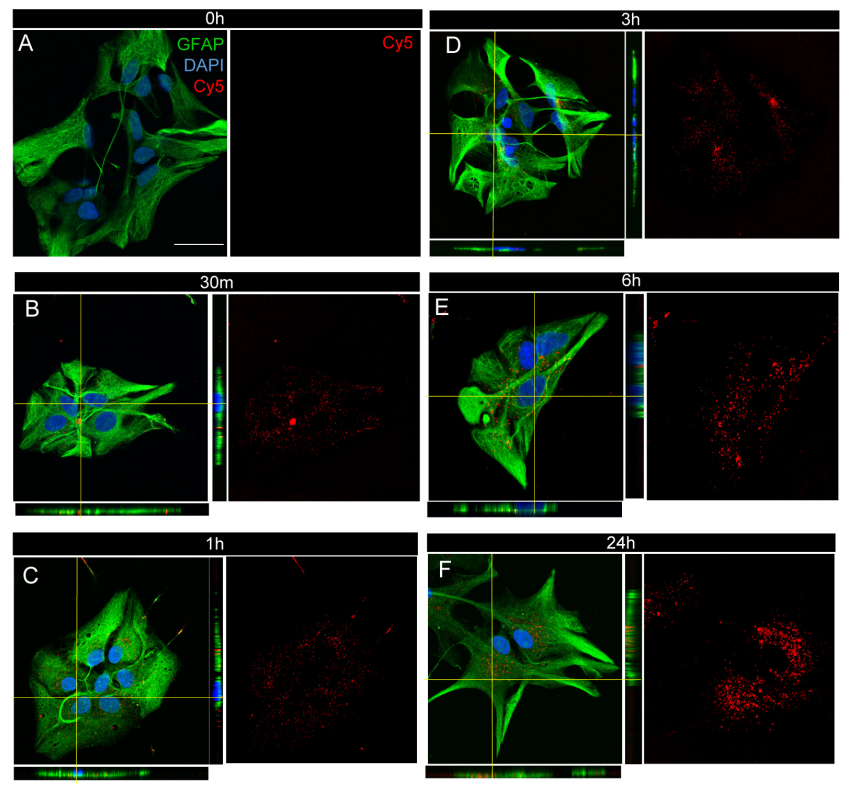

G

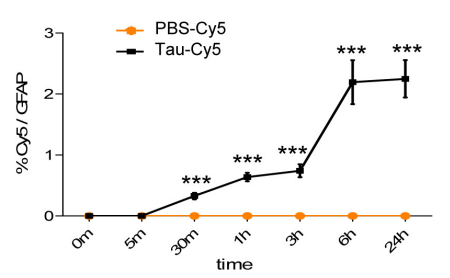

H

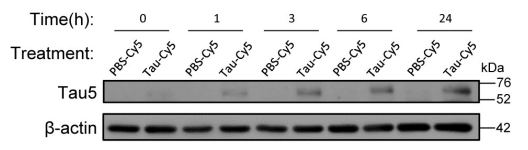

I

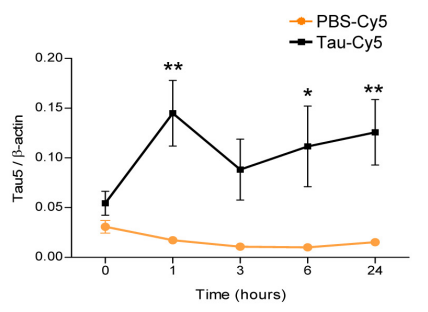

FIGURE 1 | Astrocytes internalized extracellular Tau in vitro. Astrocytes derived from primary cultures were incubated with Tau-Cy5 at different time points (A-G). (A-F) show representative immunofluorescence images of cells stained for GFAP (green) and Tau (red). Representative images of times 0 h (A), 30 min (B), $1 \mathrm{~h}$ (C), $3 \mathrm{~h}$ (D), $6 \mathrm{~h}$ (E), and $24 \mathrm{~h}$ (F) are shown. (G) shows quantification of results from immunofluorescence analysis showing \% Cy5/GFAP fluorescence intensity present in cells treated with Tau-Cy5 relative to the control PBS-Cy5. (H) western blot and (I) quantification, of Tau in cell lysates from 0 to $24 \mathrm{~h}$. Means and SE, PBS $\mathrm{O} h=0.03 \pm 0.006 ;$ Tau $0 \mathrm{~h}=0.05 \pm 0.01 ; \mathrm{PBS} 1 \mathrm{~h}=0.02 \pm 0.002 ;$ Tau $1 \mathrm{~h}=0.14 \pm 0.03 ; \mathrm{PBS} 3 \mathrm{~h}=0.01 \pm 0.002 ;$ Tau $3 \mathrm{~h}=0.09 \pm 0.03 ; \mathrm{PBS}$ $6 \mathrm{~h}=0.01 \pm 0.003$; Tau $6 \mathrm{~h}=0.11 \pm 0.04$; PBS $24 \mathrm{~h}=0.01 \pm 0.002$; and Tau $24 \mathrm{~h}=0.12 \pm 0.03$. An $\mathrm{N}=5$ independent experiments were performed. Bars show means \pm SE. $* p \leq 0.05,{ }^{* *} p \leq 0.01$, and ${ }^{* * *} p \leq 0.001$. Scale bar, $50 \mu \mathrm{m}$.

intracellular Tau forms filamentous of hyperphosphorylated and aggregated protein inside the cells that is associated with the progression of neurodegeneration (Ballatore et al., 2007; Goedert and Spillantini, 2011; Arendt et al., 2016; Medina et al., 2016). In these diseases, Tau inclusions have also been reported in glial cells, such as microglia (Odawara et al., 1995; Bolos et al., 2015; Leyns and Holtzman, 2017) and astrocytes (Kovacs et al., 2016; Ferrer et al., 2018), despite their lack of Tau expression. These observations could be explained by the internalization of Tau previously released into the extracellular space through physiological and pathological mechanisms (Simon et al., 2012; Pooler et al., 2013; Perez et al., 2016) and its posterior secretion again by neighboring cells (Perea et al., 2018b). The transfer of Tau between brain cell populations (Holmes and Diamond, 2014; Medina and Avila, 2014a; Calafate et al., 2015; Maphis et al., 2015) leads to its propagation throughout the CNS.

Although fibrillary and aggregated Tau, which are formed by hyperphosphorylated Tau, are considered a histopathological hallmark of tauopathies (Selkoe et al., 1982), monomeric forms of the protein are also involved in toxicity and the spread of the disease (Gomez-Ramos et al., 2006; Cowan and Mudher, 2013; Gerson et al., 2014; Bolos et al., 2017b; Perea et al., 2018a). In this regard, we previously reported the internalization of extracellular monomeric Tau by microglia (Bolos et al., 2015), and in the present work we confirmed the same event in astrocyte primary cultures. These results support the notion that glial cells contribute to the cell-to-cell propagation of Tau (Leyns and Holtzman, 2017; Perea et al., 2018b).

Several mechanisms of extracellular Tau internalization in neurons (Wang et al., 2017; Katsinelos et al., 2018) and glial cells have been described (Luo et al., 2015; Bolos et al., 2017a). Among them, HSPGs have been shown to be involved in the uptake of fibrillary and aggregated Tau (Holmes et al., 2013; Martini-Stoica et al., 2018; Rauch et al., 2018). However, here we have demonstrated that the uptake of monomeric Tau in astrocytes is independent of HSPGs. Both in the presence of heparin, which competes with Tau for binding to HSPGs, as well as after HSPGs removal using heparinase, the amount of internalized Tau in astrocytes was the same. This observation implies that another internalization mechanism is responsible for 


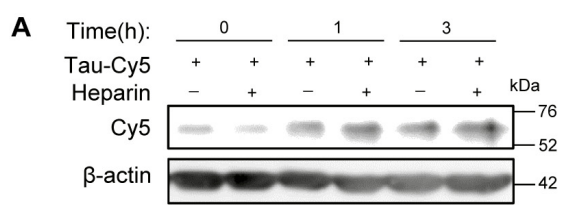

B

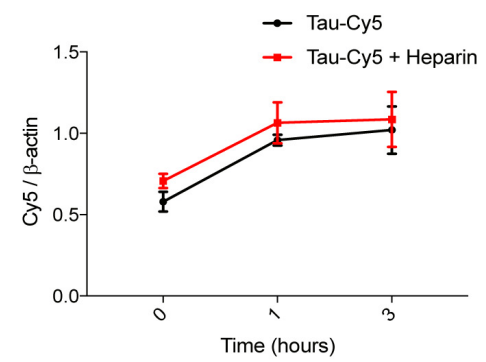

E

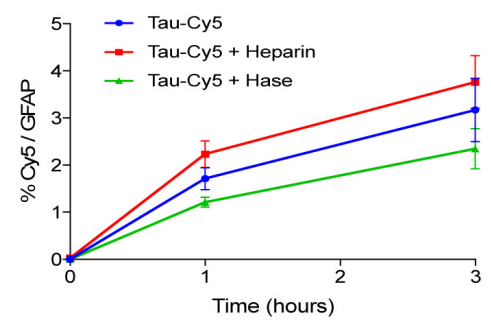

G

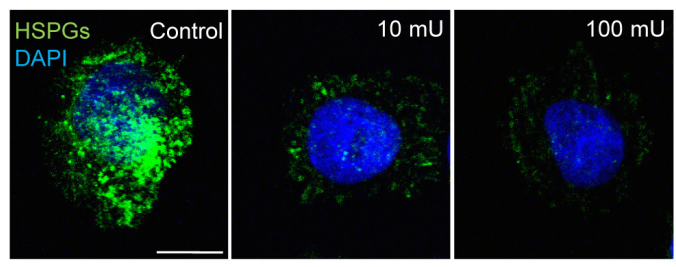

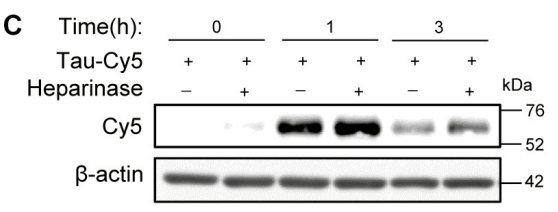

D

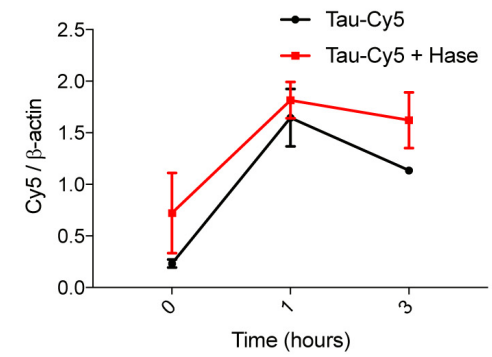

F

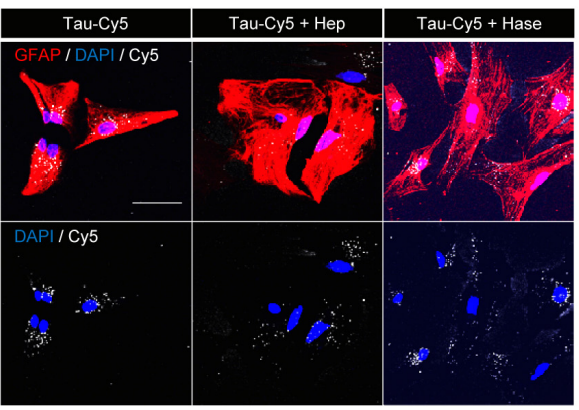

$\mathrm{H}$

I

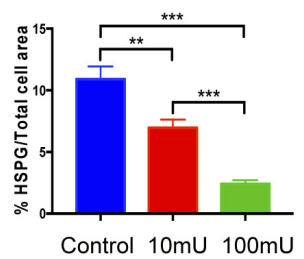

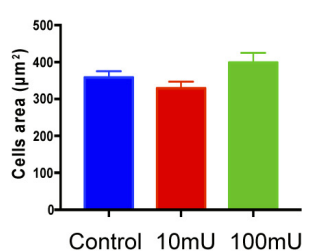

FIGURE 2 | Internalization of monomeric Tau in astrocytes is not through heparan sulfate proteoglycans. Representative western blot (A) and quantification (B) of the time course of Tau-Cy5 internalization from 0 to $3 \mathrm{~h}$ in the presence or absence of heparin. Cells were treated with Tau-Cy5 (control, $\mathrm{T}$ ) or Tau-Cy $5+$ heparin ( $+\mathrm{H}$-Hep) for different times, and Cy5 was analyzed in cell lysates. Note that the internalization of Tau did not change in the presence of heparin. Means and SE, $\mathrm{T}$ $\mathrm{O} h=0.58 \pm 0.06 ; \mathrm{T}+\mathrm{Hep} 0 \mathrm{~h}=0.71 \pm 0.04 ; \mathrm{T} 1 \mathrm{~h}=0.96 \pm 0.03 ; \mathrm{T}+\mathrm{Hep} 1 \mathrm{~h}=1.06 \pm 0.13 ; \mathrm{T} 3 \mathrm{~h}=1.02 \pm 0.15 ;$ and $\mathrm{T}+\mathrm{Hep} 3 \mathrm{~h}=1.09 \pm 0.17$. Representative western blot (C) and quantification (D) of Tau-Cy5 with ( $\mathrm{T}+\mathrm{Hase}$ ) or without heparinase treatment ( $\mathrm{T}$ ) from 0 to $3 \mathrm{~h}$. Means and SE: $\mathrm{T} 0 \mathrm{~h}=0.23 \pm 0.04$; $\mathrm{T}+\mathrm{Hase}$ $\mathrm{O} h=0.72 \pm 0.4 ; \mathrm{T} 1 \mathrm{~h}=1.65 \pm 0.28 ; \mathrm{T}+$ Hase $1 \mathrm{~h}=1.82 \pm 0.17 ; \mathrm{T} 3 \mathrm{~h}=1.13 \pm 0.03$; and T+Hase $3 \mathrm{~h}=1.62 \pm 0.27$. Note that the internalization of Tau did not change after the treatment with heparinase. Quantification of Tau-Cy5 internalization measured by immunocytochemistry (E) and some representative images of $1 \mathrm{~h}$ time point after treatment (F) confirm the results obtained by western blot. Heparinase treatment reduces HSPGs (G-I). Representative images of CHO cells treated with 0,10 , or $100 \mathrm{mU} / \mathrm{ml}$ of heparinase $\mathbf{( G )}$ and quantification of HSPGs $\mathbf{( H )}$. Means and SE, control = 10.9 \pm 1.02 ; heparinase $10 \mathrm{mU}=6.9 \pm 0.67$; and heparinase $100 \mathrm{mU}=2.4 \pm 0.29$. Quantification of the total area of the cells (I) support that heparinase treatment reduces the presence of HSPGs in the cells. An $N=3$ independent experiments were performed. Bars show means \pm SE. ${ }^{* *} p \leq 0.01$ and ${ }^{* * *} p \leq 0.001$. Scale bar, $50 \mu \mathrm{m}$ (F) and $10 \mu \mathrm{m}(\mathbf{G})$.

the uptake of monomeric Tau species by astrocytes. These results are in agreement with similar findings obtained in a recent study performed with $\alpha$-synuclein (Ihse et al., 2017). Those authors demonstrated that only aggregated $\alpha$-synuclein, and not other species of the protein, was internalized into a range of glial cells through HSPGs.

It appears that the mechanism by which fibrillary and/or aggregated Tau enters cells differs from that used by the monomeric Tau form. In this regard, monomeric Tau is internalized by muscarinic receptors M1 and M3
(Gomez-Ramos et al., 2006) in neurons; by CX3CR1 receptors in microglia (Bolos et al., 2017a); and by a non-HSPG-mediated mechanism in astrocytes. In contrast, the aggregated and fibrillary form of Tau is taken up by neurons via the HSPG pathway. These differences could be relevant for the design of potential drugs that target receptors and have the capacity to protect against the propagation of Tau in neurodegenerative diseases.

In summary, here we have shown that extracellular monomeric Tau is taken up by astrocytes. However, contrary to 
what we expected, this internalization is not HSPG-dependent. The mechanism by which monomeric Tau enters astrocytes is still unknown and deserves further attention.

\section{ETHICS STATEMENT}

This study was carried out in accordance with European Community Guidelines (directive 86/609/EEC) and handled following European and local animal care protocols. Animal experiments received the approval of the CBMSO's Ethics Committee and the National Ethics Committee (AEEC-CBMSO-62/14).

\section{AUTHOR CONTRIBUTIONS}

JD-B, JÁ, FH, and MB conceived and designed the study. JP, EL, and $\mathrm{MB}$ collected the data. JP and MB analyzed the data. MB

\section{REFERENCES}

Arendt, T., Stieler, J. T., and Holzer, M. (2016). Tau and tauopathies. Brain Res. Bull. 126, 238-292. doi: 10.1016/j.brainresbull.2016.08.018

Avila, J., Jimenez, J. S., Sayas, C. L., Bolos, M., Zabala, J. C., Rivas, G., et al. (2016). Tau Structures. Front. Aging Neurosci. 8:262.

Ballatore, C., Lee, V. M., and Trojanowski, J. Q. (2007). Tau-mediated neurodegeneration in alzheimer's disease and related disorders. Nat. Rev. Neurosci. 8, 663-672. doi: 10.1038/nrn2194

Bolos, M., Llorens-Martin, M., Jurado-Arjona, J., Hernandez, F., Rabano, A., and Avila, J. (2015). Direct evidence of internalization of tau by microglia in vitro and in vivo. J. Alzheimers Dis. 50, 77-87. doi: 10.3233/JAD-150704

Bolos, M., Llorens-Martin, M., Perea, J. R., Jurado-Arjona, J., Rabano, A., Hernandez, F., et al. (2017a). Absence of CX3CR1 impairs the internalization of Tau by microglia. Mol. Neurodegener. 12:59. doi: 10.1186/s13024-017-0200-1

Bolos, M., Pallas-Bazarra, N., Terreros-Roncal, J., Perea, J. R., Jurado-Arjona, J., Avila, J., et al. (2017b). Soluble Tau has devastating effects on the structural plasticity of hippocampal granule neurons. Transl. Psychiatry 7:1267. doi: 10.1038/s41398-017-0013-6

Braak, H., and Braak, E. (1991). Neuropathological stageing of Alzheimer-related changes. Acta Neuropathol. 82, 239-259. doi: 10.1007/bf00308809

Buee, L., and Delacourte, A. (1999). Comparative biochemistry of tau in progressive supranuclear palsy, corticobasal degeneration, FTDP-17 and Pick's disease. Brain Pathol. 9, 681-693. doi: 10.1111/j.1750-3639.1999.tb00550.x

Calafate, S., Buist, A., Miskiewicz, K., Vijayan, V., Daneels, G., de Strooper, B., et al. (2015). Synaptic contacts enhance cell-to-cell tau pathology propagation. Cell Rep. 11, 1176-1183. doi: 10.1016/j.celrep.2015.04.043

Cowan, C. M., and Mudher, A. (2013). Are tau aggregates toxic or protective in tauopathies? Front. Neurol. 4:114. doi: 10.3389/fneur.2013.00114

Drubin, D. G., and Kirschner, M. W. (1986). Tau protein function in living cells. J. Cell Biol. 103, 2739-2746. doi: 10.1083/jcb.103.6.2739

Ferrer, I., Garcia, M. A., Gonzalez, I. L., Lucena, D. D., Villalonga, A. R., Tech, M. C., et al. (2018). Aging-related tau astrogliopathy (ARTAG): not only tau phosphorylation in astrocytes. Brain Pathol. 28, 965-985. doi: 10.1111/bpa. 12593

Fox, L. M., William, C. M., Adamowicz, D. H., Pitstick, R., Carlson, G. A., SpiresJones, T. L., et al. (2011). Soluble tau species, not neurofibrillary aggregates, disrupt neural system integration in a tau transgenic model. J. Neuropathol. Exp. Neurol. 70, 588-595. doi: 10.1097/NEN.0b013e318220a658

Gerson, J. E., Castillo-Carranza, D. L., and Kayed, R. (2014). Advances in therapeutics for neurodegenerative tauopathies: moving toward the specific targeting of the most toxic tau species. ACS Chem. Neurosci. 5, 752-769. doi: $10.1021 / \mathrm{cn} 500143 \mathrm{n}$ wrote the first draft of the manuscript. All the authors read and approved the final manuscript.

\section{FUNDING}

This study was supported by the Spanish Ministry of Economy and Competitiveness [SAF-2014-53040-P (JÁ) and BFU201677885-P (FH)] and the Centro de Investigación Biomédica en Red sobre Enfermedades Neurodegenerativas (CIBERNED, Spain) (JÁ).

\section{ACKNOWLEDGMENTS}

The authors would like to thank Esther García and Raquel Cuadros for their technical assistance and the CBMSO animal and confocal microscopy facilities for their help with diverse experimental protocols.

Goedert, M., and Spillantini, M. G. (2011). Pathogenesis of the tauopathies. J. Mol. Neurosci. 45, 425-431. doi: 10.1007/s12031-011-9593-4

Gomez-Ramos, A., Diaz-Hernandez, M., Cuadros, R., Hernandez, F., and Avila, J. (2006). Extracellular tau is toxic to neuronal cells. FEBS Lett. 580, 4842-4850. doi: 10.1016/j.febslet.2006.07.078

Gomez-Ramos, A., Diaz-Hernandez, M., Rubio, A., Diaz-Hernandez, J. I., MirasPortugal, M. T., and Avila, J. (2009). Characteristics and consequences of muscarinic receptor activation by tau protein. Eur. Neuropsychopharmacol. 19, 708-717. doi: 10.1016/j.euroneuro.2009.04.006

Holmes, B. B., DeVos, S. L., Kfoury, N., Li, M., Jacks, R., Yanamandra, K., et al. (2013). Heparan sulfate proteoglycans mediate internalization and propagation of specific proteopathic seeds. Proc. Natl. Acad. Sci. U.S.A. 110, E3138-E3147. doi: $10.1073 /$ pnas. 1301440110

Holmes, B. B., and Diamond, M. I. (2014). Prion-like properties of Tau protein: the importance of extracellular Tau as a therapeutic target. J. Biol. Chem. 289, 19855-19861. doi: 10.1074/jbc.R114.549295

Hyman, B. T. (2014). Tau propagation, different tau phenotypes, and prion-like properties of tau. Neuron 82, 1189-1190. doi: 10.1016/j.neuron.2014.06.004

Ihse, E., Yamakado, H., van Wijk, X. M., Lawrence, R., Esko, J. D., and Masliah, E. (2017). Cellular internalization of alpha-synuclein aggregates by cell surface heparan sulfate depends on aggregate conformation and cell type. Sci. Rep. 7:9008. doi: 10.1038/s41598-017-08720-5

Katsinelos, T., Zeitler, M., Dimou, E., Karakatsani, A., Muller, H. M., Nachman, E., et al. (2018). Unconventional secretion mediates the trans-cellular spreading of tau. Cell Rep. 23, 2039-2055. doi: 10.1016/j.celrep.2018.04.056

Kovacs, G. G., Ferrer, I., Grinberg, L. T., Alafuzoff, I., Attems, J., Budka, H., et al. (2016). Aging-related tau astrogliopathy (ARTAG): harmonized evaluation strategy. Acta Neuropathol. 131, 87-102. doi: 10.1007/s00401-015-1509-x

Leyns, C. E. G., and Holtzman, D. M. (2017). Glial contributions to neurodegeneration in tauopathies. Mol. Neurodegener. 12:50. doi: 10.1186/ s13024-017-0192-x

Luo, W., Liu, W., Hu, X., Hanna, M., Caravaca, A., and Paul, S. M. (2015). Microglial internalization and degradation of pathological tau is enhanced by an anti-tau monoclonal antibody. Sci. Rep. 5:11161. doi: 10.1038/srep11161

Maphis, N., Xu, G., Kokiko-Cochran, O. N., Jiang, S., Cardona, A., Ransohoff, R. M., et al. (2015). Reactive microglia drive tau pathology and contribute to the spreading of pathological tau in the brain. Brain 138, 1738-1755. doi: 10.1093/brain/awv081

Martini-Stoica, H., Cole, A. L., Swartzlander, D. B., Chen, F., Wan, Y. W., Bajaj, L., et al. (2018). TFEB enhances astroglial uptake of extracellular tau species and reduces tau spreading. J. Exp. Med. 215, 2355-2377. doi: 10.1084/jem.20172158

Medina, M., and Avila, J. (2014a). Is tau a prion-like protein? J. Alzheimers Dis. 40(Suppl. 1), S1-S3. 
Medina, M., and Avila, J. (2014b). The role of extracellular Tau in the spreading of neurofibrillary pathology. Front. Cell Neurosci. 8:113. doi: 10.3389/fncel.2014. 00113

Medina, M., Hernandez, F., and Avila, J. (2016). New features about tau function and dysfunction. Biomolecules 6:E21. doi: 10.3390/biom602 0021

Medina, M., Montejo de Garcini, E., and Avila, J. (1995). The role of tau phosphorylation in transfected COS-1 cells. Mol. Cell. Biochem. 148, 79-88. doi: 10.1007/bf00929506

Odawara, T., Iseki, E., Kosaka, K., Akiyama, H., Ikeda, K., and Yamamoto, T. (1995). Investigation of tau-2 positive microglia-like cells in the subcortical nuclei of human neurodegenerative disorders. Neurosci. Lett. 192, 145-148. doi: 10.1016/0304-3940(95)11595-n

Perea, J. R., Avila, J., and Bolos, M. (2018a). Dephosphorylated rather than hyperphosphorylated Tau triggers a pro-inflammatory profile in microglia through the p38 MAPK pathway. Exp. Neurol. 310, 14-21. doi: 10.1016/j. expneurol.2018.08.007

Perea, J. R., Llorens-Martin, M., Avila, J., and Bolos, M. (2018b). The role of microglia in the spread of tau: relevance for tauopathies. Front. Cell Neurosci. 12:172. doi: 10.3389/fncel.2018.00172

Perez, M., Cuadros, R., Hernandez, F., and Avila, J. (2016). Secretion of fulllength tau or tau fragments in a cell culture model. Neurosci. Lett. 634, 63-69. doi: 10.1016/j.neulet.2016.09.026

Pooler, A. M., Phillips, E. C., Lau, D. H., Noble, W., and Hanger, D. P. (2013). Physiological release of endogenous tau is stimulated by neuronal activity. EMBO Rep. 14, 389-394. doi: 10.1038/embor.2013.15

Rauch, J. N., Chen, J. J., Sorum, A. W., Miller, G. M., Sharf, T., See, S. K., et al. (2018). Tau internalization is regulated by 6-o sulfation on heparan sulfate proteoglycans (HSPGs). Sci. Rep. 8:6382. doi: 10.1038/s41598-018-24904-z

Selkoe, D. J., Ihara, Y., and Salazar, F. J. (1982). Alzheimer's disease: insolubility of partially purified paired helical filaments in sodium dodecyl sulfate and urea. Science 215, 1243-1245. doi: 10.1126/science.6120571
Simon, D., Garcia-Garcia, E., Royo, F., Falcon-Perez, J. M., and Avila, J. (2012). Proteostasis of tau. Tau overexpression results in its secretion via membrane vesicles. FEBS Lett. 586, 47-54. doi: 10.1016/j.febslet.2011.11.022

Spires-Jones, T. L., Stoothoff, W. H., de Calignon, A., Jones, P. B., and Hyman, B. T. (2009). Tau pathophysiology in neurodegeneration: a tangled issue. Trends Neurosci. 32, 150-159. doi: 10.1016/j.tins.2008.11.007

Stopschinski, B. E., Holmes, B. B., Miller, G. M., Manon, V. A., Vaquer-Alicea, J., Prueitt, W. L., et al. (2018). Specific glycosaminoglycan chain length and sulfation patterns are required for cell uptake of tau versus alpha-synuclein and beta-amyloid aggregates. J. Biol. Chem. 293, 10826-10840. doi: 10.1074/jbc. RA117.000378

Wang, Y., Balaji, V., Kaniyappan, S., Kruger, L., Irsen, S., Tepper, K., et al. (2017). The release and trans-synaptic transmission of Tau via exosomes. Mol. Neurodegener. 12:5. doi: 10.1186/s13024-016-0143-y

Weingarten, M. D., Lockwood, A. H., Hwo, S. Y., and Kirschner, M. W. (1975). A protein factor essential for microtubule assembly. Proc. Natl. Acad. Sci. U.S.A. 72, 1858-1862. doi: 10.1073/pnas.72.5.1858

Yamada, M., and Hamaguchi, T. (2018). The sulfation code for propagation of neurodegeneration. J. Biol. Chem. 293, 10841-10842. doi: 10.1074/jbc.H118. 003970

Conflict of Interest Statement: The authors declare that the research was conducted in the absence of any commercial or financial relationships that could be construed as a potential conflict of interest.

Copyright (c) 2019 Perea, López, Díez-Ballesteros, Ávila, Hernández and Bolós. This is an open-access article distributed under the terms of the Creative Commons Attribution License (CC BY). The use, distribution or reproduction in other forums is permitted, provided the original author(s) and the copyright owner(s) are credited and that the original publication in this journal is cited, in accordance with accepted academic practice. No use, distribution or reproduction is permitted which does not comply with these terms. 